\author{
ANNALS OF “DUNAREA DE JOS” UNIVERSITY OF GALATI \\ MATHEMATICS, PHYSICS, THEORETICAL MECHANICS \\ FASCICLE II, YEAR XI (XLII) 2019, No. 1
}

Article DOI: $\underline{\text { https://doi.org/10.35219/ann-ugal-math-phys-mec.2019.1.03 }}$

\title{
AN OPTIMAL SOLUTION BASED ON IMAGE FUSION FOR BLOOD VESSEL DIAMETER MEASUREMENTS IN CEREBRAL ANGIOGRAPHIES
}

\author{
Cristian-Dragoș Obreja ${ }^{1}$, Luminiţa Moraru ${ }^{2}$
I*Department for Informatics and Digital Communications, "Dunărea de Jos" University of Galati, Romania, e- mail: cobreja@ugal.ro \\ ${ }^{2}$ Faculty of Science and Environment, Physics Department, "Dunărea de Jos" University of Galati , Romania
}

\begin{abstract}
The main goal of this study is an accurate extraction of the brain vascular structure to improve detection and to enhance the blood vessels in the human brain. Data provided by Canny and Frangi edge detection algorithms are evaluated and then these disparate data are combined with a fusion algorithm for comparison purposes. This investigation is performed in an edge detection framework. The performance of the proposed methods is evaluated by conducting measurements on the diameter of the brain blood vessels in cerebral angiography images. The average diameters of the blood vessels provided by Frangi, Canny and the fusion algorithms are measured. To highlight the improvements brought by data fusion, an objective evaluation measure based on edge structural similarity and error rate have been made. Also, the superiority of the fusion algorithm in terms of image quality is proved.
\end{abstract}

Keywords: image fusion, Canny filter, Frangi filter, edge-detection, ESSIM.

\section{INTRODUCTION}

The role of the medical imaging is to improve the detection accuracy of certain vascular system disorders, such as cerebral vascular accidents, hypertension or diabetic retinopathy. In order to detect vascular anomalies a database of medical digital images was used, but the existence of complex background, image artifacts, the vascular system asymmetry and uneven illumination lead to a certain degree of inaccuracy of the measurements. Due to this reason, different segmentation and edge detection techniques were developed and improved over the past decades [1-4]. These algorithms used different types of gradient masks and morphological operators to generate accurate vascular maps. Furthermore, some segmentation methods used a threshold to split the image content into foreground and background [4-9], either for the entire image or from some localized areas of the image in the case of more advanced method. In the medical images case, the main drawback faced by these algorithms is generated by the existence of the background noise, uneven illumination and artifacts. To overcome these disadvantages, a method that fuses images in pairs of two was proposed. This technique is based on Dempster-Shafer (DS) mathematical theory of evidence and combines the pre-segmented medical images [10,11].

In this study, we asses our proposed image technique in comparison with two established edge-detection algorithms. The brain vessels are detected using the Canny and Frangi methods, and the results generated by them are fused using the Dempster-Shafer method. The quality of the images generated by Canny and Frangi algorithms and the fused images is improved using so called ,contrast limited adaptive histogram equalization technique'. These improved images are used to measure the 
blood vessel diameters and to estimate the error rate of this method. The accuracy of the proposed vascular edge detection algorithm was evaluated using the edge strength similarity-based image quality metric (ESSIM) technique that analyzes the detected edges from the reference and processed images. This paper is organized as follows: section 2 presents the materials and methods, section 3 contains the experimental results and subsequent discussion and conclusions are shown in the final section.

\section{MATERIALS AND METHODS}

\section{Database and the Algorithm}

A database containing 17 images from the "The Whole Brain Atlas" (WBA) website from Harvard Medical School [12] has been analyzed. The database consists of 10 randomly selected images that belong to healthy patients and 7 images of cerebral vascular accident. The chart of the method used in this study is presented in figure 1 .

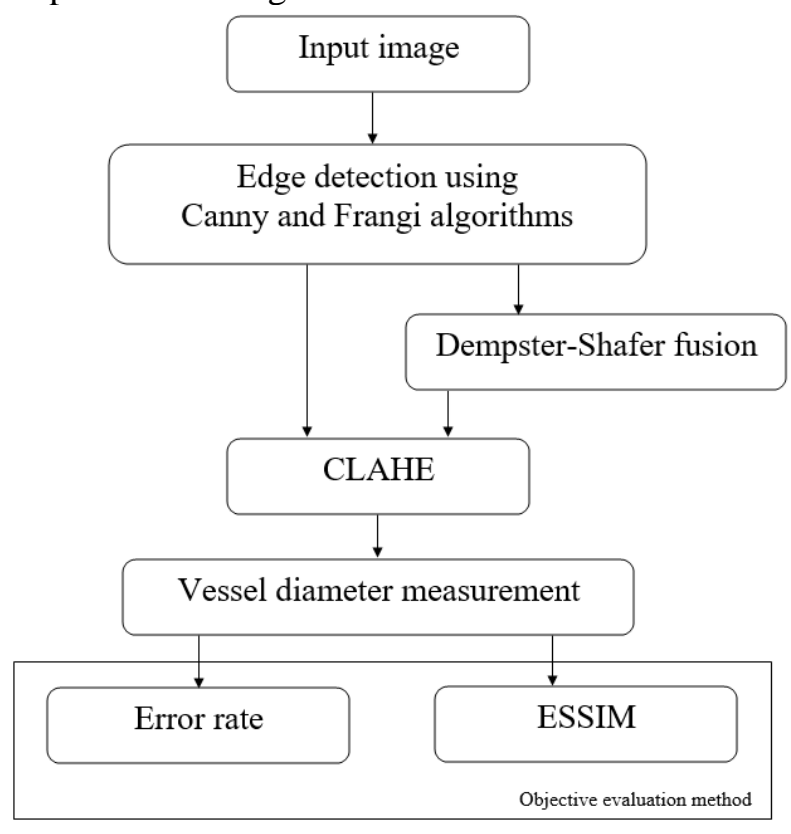

Fig. 1. Flow chart of the proposed method

\section{Canny filter}

The Canny filter method is considered an optimal edge detector as it provides the lowest error rate regarding the edges found in the image [13]. Furthermore, it generates a low response to the nonedge pixels. At the same time, it preforms smoothing and de-noising operations using a standard convolution mask: $[2,4,5,4,2 ; 4,9,12,9,4 ; 5,12,15,12,5 ; 4,9,12,9,4 ; 2,4,5,4,2]$. The Canny filter returns the gradient value as follows:

$$
G=\sqrt{\left(\partial_{x} I(x, y)\right)^{2}+\left(\partial_{y} I(x, y)\right)^{2}}, \theta=\arctan \left(\frac{G_{y}}{G_{x}}\right)
$$

were the first derivatives in the horizontal and vertical directions are $G_{x}=\left(\partial_{x} I(x, y)\right)^{2}$ and $G_{y}=\left(\partial_{y} I(x, y)\right)^{2}$.

To determine the edge strength, the gradient $G$ is computed and those pixels that do not belong to a local maximum on the gradient direction are dismissed from the edge [13,14]. Furthermore, two thresholds are used T1 and T2. They detect the weak and strong blood vessel edges, but the main disadvantage is that the low threshold (T1) generates false edges by interpreting image artifacts as edges. [14]. In the current study, the threshold values were experimentally set to $\mathrm{T} 1=0.002$ and $\mathrm{T} 2=$ 0.019 .

\section{Frangi filter}


This method of edge detection computes the Hessian matrix and gathers data from the Hessian eigenvalues. The image matrix is determined using the Gaussian second order derivatives and the Frangi edge detector works in two steps [15]. In the first step, the filter compares the blood vessels with the shape of an ideal tube using the following algorithm:

$$
v_{F}^{\sigma}(x)=\left\{\left(1-\exp \left(-\frac{R_{A}^{2}}{2 \alpha^{2}}\right)\right) \exp \left(-\frac{R_{B}^{2}}{2 \beta^{2}}\right)\left(1-\exp \left(-\frac{S^{2}}{2 c^{2}}\right)\right),\right. \text { otherwise }
$$

where $\alpha, \beta, c$ are parameters used to control sensitivity and $\lambda_{1}, \lambda_{2}, \lambda_{3}$ represent the eigenvalues of the Hessian matrix. In the second step, a comparative study between blob structures on one side and tube and plate structures is made using the following three measures [16,17]:

$$
\begin{gathered}
R_{A}=\frac{\left|\lambda_{2}\right|}{\left|\lambda_{3}\right|}=\frac{(\text { the surface of the largest section }) / \pi}{(\text { the } \text { half }- \text { length of the longest axis })^{2}} \\
R_{B}=\frac{\left|\lambda_{1}\right|}{\sqrt{\left|\lambda_{2} \lambda_{3}\right|}}=\frac{(\text { volume }) /\left(\frac{4 \pi}{3}\right)}{(\text { the largest cross section })^{3 / 2}} \\
s=\sqrt{\lambda_{1}^{2}+\lambda_{2}^{2}+\lambda_{3}^{2}}
\end{gathered}
$$

where $R_{A}$ is the dissimilarity values between tube and plate structures, $R_{B}$ are the blob structures and $S$ is the background noise. Furthermore, the response is integrated if it fulfills the conditions of a being a tube, at scales between minimum and maximum: $v_{F}(x)=\max _{\sigma_{\min } \leq \sigma \leq \sigma_{\max }} v_{F}^{\sigma}(x)$.

\section{Dempster-Shafer Fusion for Edge Detection}

The fusion edge detection algorithm was used to overcome the recognized weaknesses of the independent methods and to detect the real blood vessels. This method combines information based on the mathematical theory of evidence [18,19]. The bodies of evidence used in the fusion method are represented by the results generated from Canny and Frangi filters. Also, the part of necessary information is obtained from the mass function: $\mathrm{n}_{\mathrm{i}, 2}=\mathrm{P}(\varphi$ loff - edge $) ; \mathrm{n}_{\mathrm{i}, 2}=\mathrm{P}(\varphi$ loff - edge $)$, with $i=$ $1,2,3$. The proposed fusion method combines different trust functions, according to the following equation [20]:

$$
n_{i \oplus j}(A)=\frac{\sum_{B \cap C=A} n_{i}(B) n_{j}(C)}{1-\sum_{B \cap C=\emptyset} n_{i}(B) n_{j}(C)} \text { when } A \neq \emptyset
$$

where $n_{i}$ and $n_{j}$ are the bodies of evidence; $A, B$ and $C$ are the event sets generated by the fusion method, $n_{i \oplus j}(\emptyset)=0$ is used to discard the conflicts generated by the edge detector and $i, j=1,2$.

Furthermore, because the classic edge detection methods generate results that are on-edges (E) or off-edges $(\mathrm{N})$ pixels, the DS fusion rule has the following equation:

$$
n(E)=\frac{w n_{i}(E) \cdot w n_{j}(E)}{1-w n_{i}(E) \cdot(1-w) n_{j}(N)-(1-w) n_{i}(N) \cdot w n_{j}(E)}
$$

where, $n(E)$ is the edge confidence, $n(N)$ is the non-edges confidence and $w$ is the weighted DS evidence combination for optimization purposes. The confidence map is generated, and the edge magnitude is computed using $M_{\text {edge }}=n_{\varphi} n(E)$, where $\varphi$ represents Canny and Frangi filters $[21,22]$.

\section{CLAHE (Contrast-Limited Adaptive Histogram Equalization)}

In order to detect the finer blood vessel edges, the contrast has been improved based on the local histogram manipulation. CLAHE was applied on both the fused images and Canny and Frangi filtered images. This method performs an equalization of the histogram and a contrast improvement based on the local histogram. Furthermore, to prevent noise over-enhancement and lower edgeshadowing, a unification of the neighboring regions is made. Thus, the bins number and contrast factor are limited and the image histogram is maintained inside the clip limit. [23, 24]. 


\section{Vessel diameter measurement and analysis}

By measuring the diameter of the blood vessels in the brain, the accuracy of the fusion method against the Canny and Frangi edge detectors was evaluated. The vessel diameters are measured using a personal method in Matlab, that detects a cross-sectional intensity profile and generates three perpendicular lines over the blood vessel. The width of the vessel is determined for all three measurements, and the diameter is determined using a linear interpolation operation.

In order to assess the fusion performance for real edges detection, the edge-based structural similarity (ESSIM) is computed. ESSIM analyzes the structural similarity of images based on the detected edges between Canny, Frangi and fusion images. For each pixel, an edge vector containing the edge amplitude and direction is built. All these edge vectors form the edge map of the image [25, 26]. The edge comparison measure, $e(R, F)$, is computed and this edge information is correlated with both, luminance $l(R, F)$ and contrast $c(R, F)$ in the ESSIM algorithm:

$$
\operatorname{ESSIM}(R, F)=[l(R, F)]^{\alpha}[c(R, F)]^{\beta}[e(R, F)]^{\gamma}
$$

where parameters $\alpha, \beta$ and $\gamma$ are used to adjust components by their own relative importance.

Furthermore, the error rate is computed for the vessels diameter measured values:

$$
E R=\frac{\left|M_{R}-M_{F}\right|}{M_{R}} \times 100 \%
$$

where, $M_{R}$ represents the original values for vessel diameter and $M_{F}$ are the values computed for the Canny, Frangi and fused images.

\section{RESULTS AND DISCUSSION}

The accuracy assessment of the DS fusion algorithm relative to the classic edge-detectors and the original images was made by measuring the diameters on 17 randomly selected angiographies.

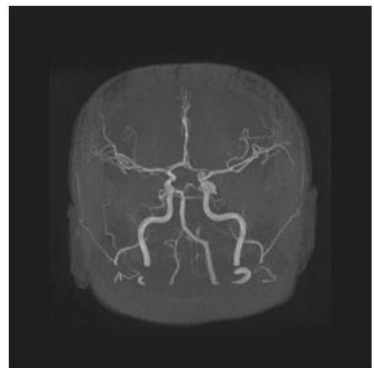

$\left(\mathrm{a}_{\mathrm{i}}\right)$

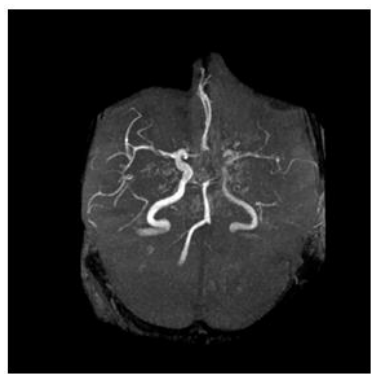

$\left(b_{\mathrm{i}}\right)$

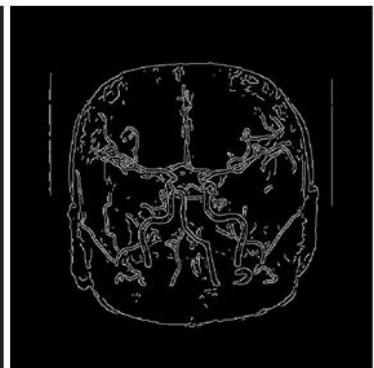

$\left(a_{j}\right)$

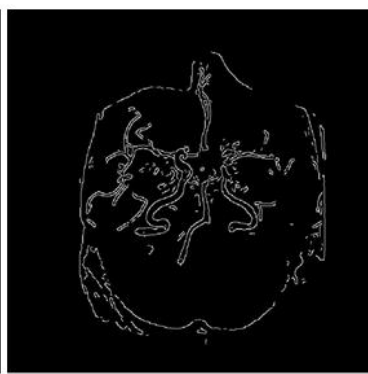

$\left(b_{j}\right)$

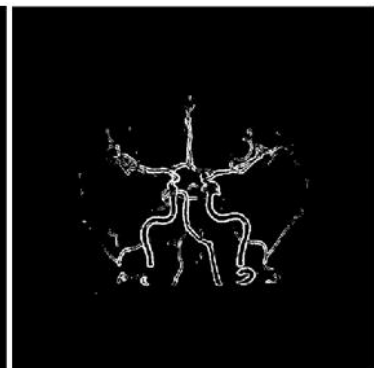

$\left(a_{k}\right)$

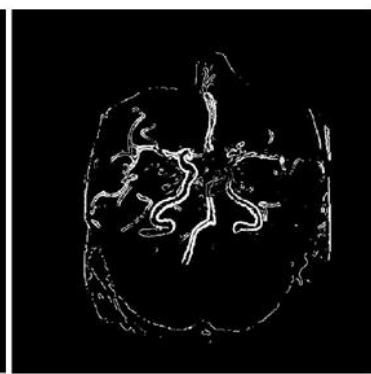

$\left(b_{\mathrm{k}}\right)$

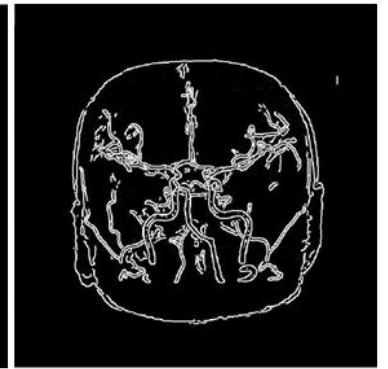

$\left(a_{1}\right)$

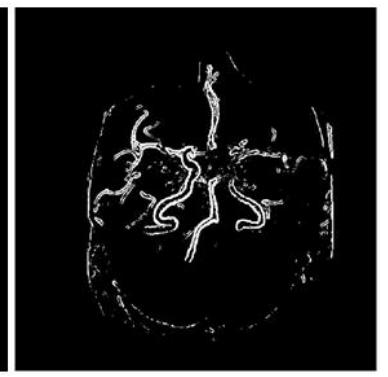

$\left(b_{1}\right)$

Fig. 2. Examples of brain vascular map.

Line 1 is for Healthy patient: $\left(a_{i}\right)$ Original input image; $\left(a_{j}\right)$ Canny filter; $\left(a_{k}\right)$ Frangi filter; (al) Dempster-Shafer fusion. Line2 is for Cerebral aneurysm: $\left(b_{i}\right)$ Original input image; $\left(b_{j}\right)$ Canny filter; $\left(b_{k}\right)$ Frangi filter; $\left(b_{l}\right)$ Dempster-Shafer fusion.

Examples of brain vascular maps for Canny, Frangi and Dempster-Shafer methods are presented in figure 2. A weight coefficient of $w=0.39$ for the Dempster-Shafer evidence combination 
was determined based on trial and error results. The fusion algorithm was applied for healthy and cerebral aneurysm affected patients.

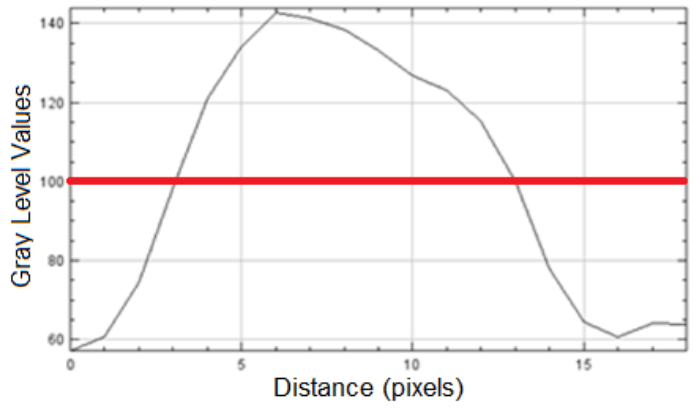

$\left(a_{i}\right)$

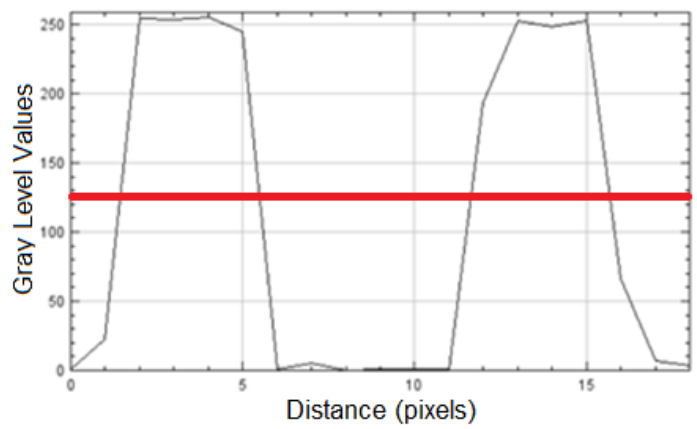

(ak)

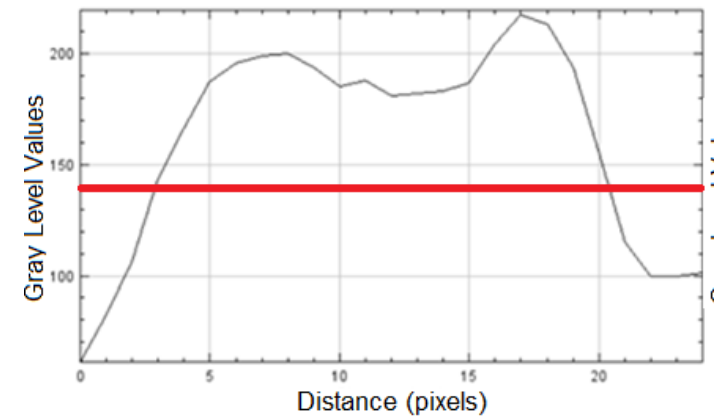

(bi)

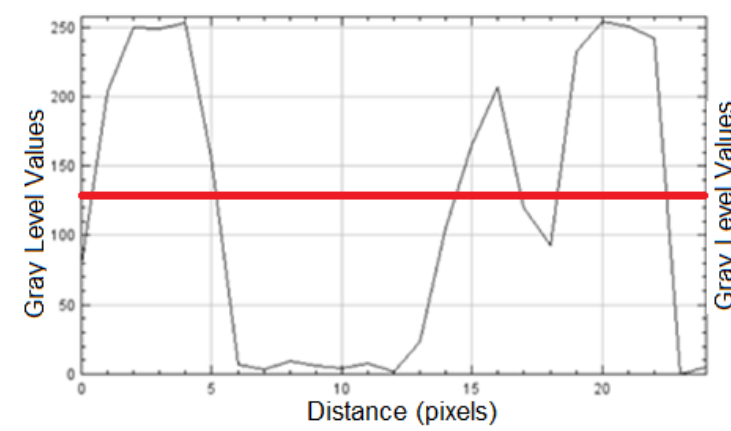

(bk)

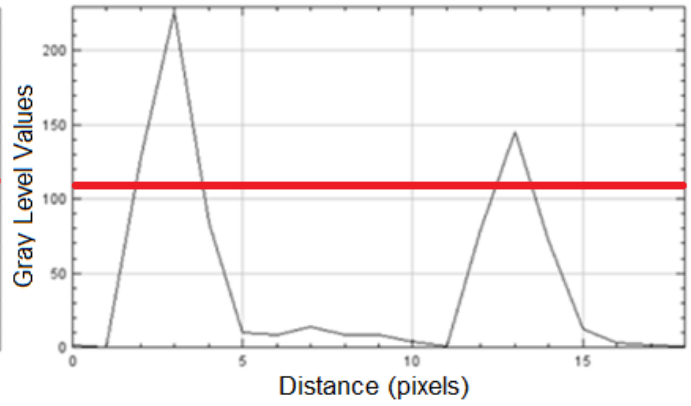

$\left(a_{j}\right)$

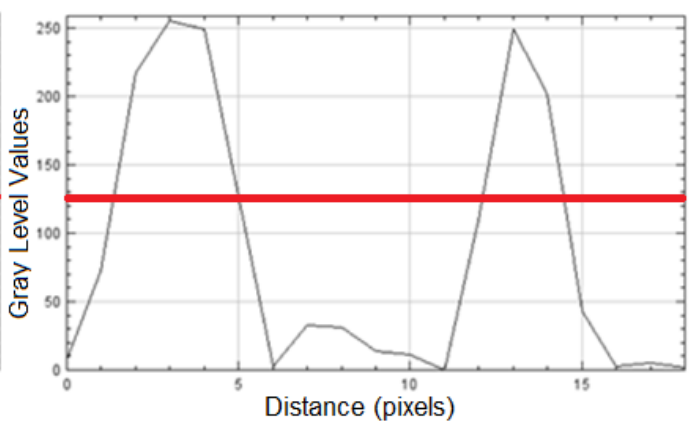

(al)

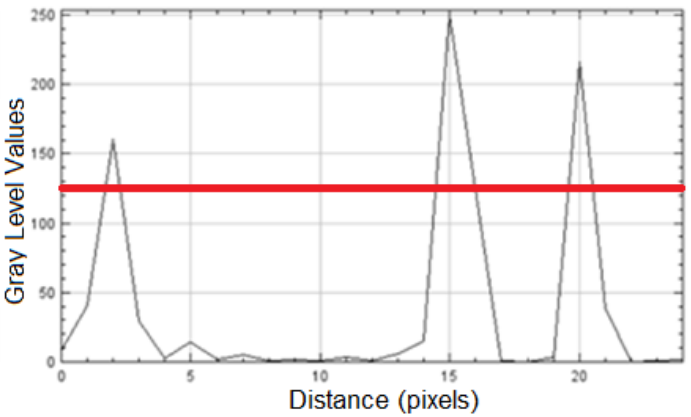

$\left(b_{j}\right)$

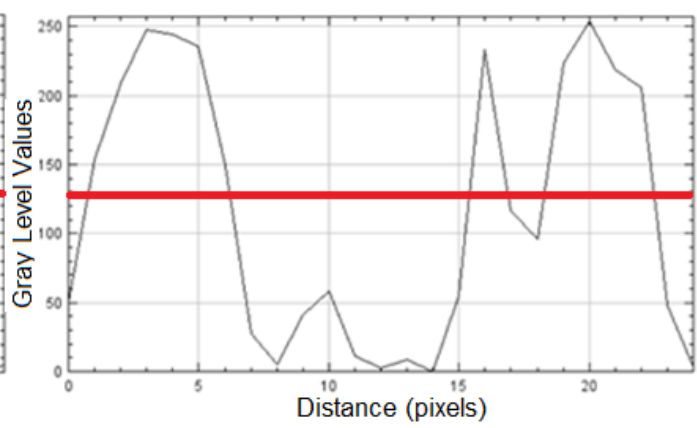

(bl)

Fig. 3. Vessel diameter estimation based on cross-sectional intensity profile.

Healthy patient: ( $\left.a_{i}\right)$ Original input image; $\left(a_{j}\right)$ Canny filter; $\left(a_{k}\right)$ Frangi filter; (al) Dempster-

Shafer fusion. Cerebral aneurysm: $\left(b_{i}\right)$ Original input image; $\left(b_{j}\right)$ Canny filter;

$\left(b_{k}\right)$ Frangi filter; $\left(b_{l}\right)$ Dempster-Shafer fusion.

Figures $3\left(a_{i}-b_{i}\right)$ depict that the original images cross-section has a single peak shape. The cross-section has this shape, because the images are gray, the pixels in background are black and in the foreground are white. Whereas, the rest of the figures demonstrates that the cross-section of any of the 
fused images has a double-valley shape because all edges consist of black pixels, and white pixels on a background. The horizontal line is drawn at half the maximum intensity peak height and its width allows for vessel diameter estimation.

Table 1 . The average vessel diameter values and the average percentage error, for healthy $(\mathrm{H})$ and cerebral aneurysm (CA), for Canny filter (CF), Frangi filter (FF), and Dempster-Shafer fusion (DS)

\begin{tabular}{|llllllll|}
\hline & Original image & $\mathbf{C F}$ & $\boldsymbol{e}_{\mathrm{CF}} \%$ & $\mathbf{F F}$ & $\boldsymbol{e}_{\mathrm{FF}} \boldsymbol{\%}$ & $\mathbf{D S}$ & $\boldsymbol{e}_{\boldsymbol{D} \boldsymbol{S}} \boldsymbol{\%}$ \\
\hline $\mathbf{H}$ & 11.84 & 11.97 & 5.34 & 12.95 & 9.42 & 12.42 & 4.96 \\
\hline $\mathbf{C A}$ & 20.01 & 18.74 & 6.35 & 22.04 & 10.14 & 21.08 & 5.35 \\
\hline
\end{tabular}

According to the values presented in the table 1, the fused images generate the lowest error rate. In table 2, the values of the structural similarity index (ESSIM) are presented. The values generated by the fused images are higher than the ones for the Canny and Frangi filters. The perfect edge value for ESSIM is 1.

Table 2. Comparison of average ESSIM for normal and cerebral aneurysm

\begin{tabular}{|lccc|}
\hline & CF & FF & DS \\
\hline H & 0.9473 & 0.9465 & 0.9562 \\
\hline CA & 0.9479 & 0.9471 & 0.9576 \\
\hline
\end{tabular}

The main goal of this research was to get an accurate vascular maps by using a image fusion method based on Dempster-Shafer theory of evidence. The first observation about the proposed method is that it detects continuous edges. The accuracy of this method was evaluated by means of the error rate of the vessels diameter measurement values and by an objective quality assessment measure, namely ESSIM.

The average diameter and error rate values for the original, fused and classic alghoritms studied angiographies are computed. According to the obtained results, between the two classic edge detection methods, Canny filter (CF) generated lower error rates for both healthy and cerebral aneurysm affected patients. The CF error was $5.34 \%$ for healthy subjects and $6.35 \%$, cerebral aneurysm, compared to $9.42 \%$ and $10.14 \%$ for FF. Furthermore, by analyzing the DS fusion method error rates, we observe that it generates a lower error rate than either one of the classic edge detectors. Also, after analyzing fig. 2 we conclude that while CF and FF, generate good results, they can often lead to false or doubled edges and also edge discontinuities. Unlike the classic filters, the fusion algorithm produced better vascular maps and lower error rates.

Furthermore, the filters used in his study are analyzed using an edge-based structural similarity (ESSIM) algorithm. The highest edge similarity and the most accurate vascular map is generated by DS fusion for healthy subjects and also for cerebral aneurysm affected patients. Analyzing the obtained results, we observe that the similarity between the classic edge detectors generated and original images is around 6\% and for DS fusion is less than 5\%. The reported results show that the edge maps generated by $\mathrm{CF}$ and FF are not accurate enough and fail to extract some edge structures from the studied angiographies. Also, the fusion method finds the middle ground between $\mathrm{CF}$ and FF and generates more continuous edges. Also, the ESSIM values for cerebral aneurysm are a little higher than those of healthy subjects, because of the distorted blood vessels. Furthermore, the DS fusion algorithm generates more accurate true edges than both Frangi and Canny filters. For both healthy and cerebral aneurysm affected patients, the DS fusion between the output images of CF and FF, generates edge maps with a higher accuracy rate than any of them used alone.

\section{CONCLUSIONS}

This study presented a comparison between the results generated by Canny and Frangi filters and those generated by DS fusion algorithm that merges the CF and FF results. According to the results, the accuracy of the vascular edge maps generated by using DS fusion is improved. The fusion performance was evaluated by meassuring the vessel diameter and computing its error rate, and by analyzing the structural similarity of the edge maps. The results indicate that DS is an effective alternative for classic filters in generating accurate vascular edge maps. 


\section{References}

1. S. Bortolin Jr., D. Welfer, International Journal of Computer Science \& Information Technology 5(5), 21-37, 2013.

2. S.S.R. Dhanushkodi, V. Manivannan, Applied Medical Informatics 33(3), 1-11, 2013.

3. B. Zhang, L. Zhang, F. Karray, Computers in Biology and Medicine 40(4), 438-445, 2009.

4. K.K. Delibasis, A.I. Kechriniotis, C. Tsonos, N. Assimakis, Computer Methods and Programs in Biomedicine 100(2), 108-122, 2010.

5. M.E. Martinez-Perez, A.D. Hughes, S.A. Thom, A.A. Bharath, K.H. Parker, Segmentation of blood vessels from red-free and fluorescein retinal images, Medical Image Analysis 11(4), 4761, 2007.

6. B. Al-Diri, A. Hunter, D. Steel, An active contour for segmenting and measuring retinal vessel, IEEE Trans. on Medical Imaging 28(9), 1488-1497, 2010.

7. C.A. Lupascu, D. Tegolo, E. Trucco, IEEE Trans. on Information Technology in Biomedicine 14(5), 1267-1274, 2010.

8. L. Zhou, M.S. Rzeszotarski, L.J. Singerman, J.M. Chokreff, IEEE Trans. on Medical Imaging 13(4), 619-626, 1994.

9. L. Moraru, C.D. Obreja, Retinal vessel enhancement based on Gaussian function, AIP Conference Proceedings 1796:040007-1, 2017. doi:10.1063/1.4972385.

10. X. Li, W.G. Wee, Retinal vessel detection and measurement for Computer aided Medical Diagnosis, J Digit Imaging 27, 120-132, 2014.

11. M. Arif, T. Brouard, N. Vincent, A fusion methodology based on Dempster-Shafer evidence theory for two biometric applications, 18th International Conference on Pa<ern Recognition 4, 590-593, 2006. doi:10.1109/ICPR.2006.68.

12. The Whole Brain Atlas, Harvard Medical School, http://www.med.harvard.edu/AANLIB/home.html.

13. J. Canny, A computational approach to edge detection, IEEE Transactions on Pattern Analysis and Machine Intelligence 8 (6), 679-698, 1986.

14. P.R. Possa, S.A. Mahmoudi, N. Harb, C. Valderrama, P. Manneback, A Multi-Resolution FPGA-Based Architecture for Real-Time Edge and Corner Detection, IEEE Transactions on Computers 63 (10), 2376-2388, 2014.

15. A. Frangi, W. Niessen, K. Vincken, M. Viergever, Multiscale vessel enhancement filtering, Medical Image Computing and Computer-Assisted Interventation MICCAI 98 130-137, 1998.

16. D. Jimenez-Carretero, A. Santos, S. Kerkstra, R.D. Rudyanto, M.J. Ledesma-Carbayo, 3D Frangi-based lung vessel enhancement filter penalizing airways, 2013 IEEE 10th International Symposium on Biomedical Imaging: From Nano to Macro San Francisco, CA, USA, April 711, 2013.

17. N. Memari, A.R. Ramli, M.I.B. Saripan, S. Mashohor, M. Moghbel, Journal of Medical and Biological Engineering, Retinal Blood Vessel Segmentation by Using Matched Filtering and Fuzzy C-means Clustering with Integrated Level Set Method for Diabetic Retinopathy Assessment, 1-19, 2018. doi.org/10.1007/s40846-018-0454-2

18. G. Shafer, Perspectives on the theory and practice of belief functions, International Journal of Approximate Reasoning 4, 323-362, 1990.

19. J. Dezert, Z. Liu, G. Mercier, Edge Detection in Color Images based on DSmT, Proceedings of the 14th International Conference on Information Fusion (FUSION), 1-8, 2011.

20. H.Wu, M. Siegel, R. Stiefelhagen, J. Yang, Sensor Fusion using Dempster-Shafer Theory, Proceedings of the 19th IEEE Instrumentation and Measurement Technology Conference (IMTC/2002) 1, 7-12, 2002.

21. T.C. Lin, Partition belief median filter based on Dempster-Shafer theory for image processing, Pattern Recognition 41(1), 139-151, 2008.

22. L. Moraru, C.D. Obreja, Nilanjan Dey, Amira S. Ashour, Soft Computing In Medical Image Analysis, Chapter: Dempster-Shafer Fusion for Effective Retinal Vessels' Diameter Measurement, (Nilanjan Dey, Amira S. Ashour, Fuqian Shi, Valentina E. Balas), Elsevier, Academic Press, pp. 149-163, 2018.ISBN: 9780128130872, 
https://www.elsevier.com/books/soft-computing-based-medical-image-analysis/dey/978-0-12813087-2

23. H.S.S. Ahmed, J. Nordin, Improving Diagnostic Viewing of Medical Images using Enhancement Algorithms, Journal of Computer Science 7(12), 1831-1838, 2011.

24. I.K. Maitra, S. Nag, S.K. Bandyopadhyay, Computer methods and programs in biomedicine 107, 175-188, 2012.

25. G.H. Chen, C.L. Yang, L.M. Po, S.L. Xie, Edge-based structural similarity for image quality assessment, IEEE International Conference on Acoustics, Speech and Signal Processing 2, 933-936, 2006.

26. S. Betrabet, C.K. Bhogayta, Structural Similarity based Image Quality Assessment using full reference method, International Journal of Scientific Engineering and Technology 4(4), 252$255,2015$. 